REVIEW ARTICLE

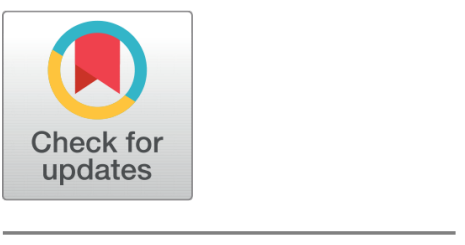

OPEN ACCESS

Received: 21.07.2020

Accepted: 14.09 .2020

Published: 03.10 .2020

Editor: Dr. Natarajan Gajendran

Citation: Simi KG, Susanth SG, Sumod SG (2020) On the vector plasma drift measurements over Equatorial lonosphere using HF Doppler radar - A brief review. Indian Journal of Science and Technology 13(35): 3642-3651. https ://doi.org/10.17485/IJST/v13i35.1084

* Corresponding author.

Tel: 9895415006

kg.simi@vtmnsscollege.ac.in

Funding: None

Competing Interests: None

Copyright: $₫ 2020$ Simi et al. This is an open access article distributed under the terms of the Creative Commons Attribution License, which permits unrestricted use, distribution, and reproduction in any medium, provided the original author and source are credited.

Published By Indian Society for Education and Environment (iSee)

ISSN

Print: 0974-6846

Electronic: 0974-5645

\section{On the vector plasma drift measurements over Equatorial lonosphere using HF Doppler radar - A brief review}

\author{
K G Simi ${ }^{1 *}$, S G Susanth ${ }^{2}$, S G Sumod ${ }^{3}$ \\ 1 Department of Physics, VTM NSS College, Dhanuvachapuram, Thiruvananthapuram, \\ 695503, Kerala, India. Tel.: 9895415006 \\ 2 Department of Applied Science and Humanities, Lourdes Matha College of Science and \\ Technology, Thiruvananthapuram, Kerala, India \\ 3 Space Science Group, Sacred Heart College, Mahatma Gandhi University, Kochi, 682013, \\ Kerala, India
}

\section{Abstract}

Background/ Objective: University of Kerala, Thiruvananthapuram ( $8.5^{\circ} \mathrm{N}, 77$ ${ }^{\circ} \mathrm{E}$, dip $0.5^{\circ} \mathrm{N}$ ) in India had a lively Space Physics research group since 1982, owing to proximity of the location to the geomagnetic dip equator. In 1982, a single frequency HF Doppler radar, operational at $5.5 \mathrm{MHz}$ was installed in the University which provided many invaluable scientific results regarding the night time ionospheric F region. In 2003, it was upgraded to a new multifrequency HF Doppler Radar thereby enabling detailed examination of vertical plasma drift measurements at three different altitudes of F-region, in a nearsimultaneous manner. Methods: This study is basically a brief review of major results of $\mathrm{F}$-region electrodynamics over Indian longitudinal sector obtained exclusively using HF Radar data. Findings: This review brings all the scientific results obtained using HF Radar about vertical drift studies over equatorial latitude and found that nearly about 40 research papers have been published in reputed national and international journals using the Radar data. Novelty: In a complementary point of view, this gives an opportunity to revisit and remember the important contributions of many of the earlier Indian scientists, who enriched ionospheric science using this unique facility.

Keywords: HF Doppler Radar; lonosphere; Vertical drift

\section{Introduction}

As is well-known, the ionosphere is a dynamical system having different layers and exhibits various types of plasma motions. The ionospheric electric fields and plasma drifts over equatorial/low latitude regions are quite complex due to the highly varying $\mathrm{E}$ and F-region electrodynamical processes. It is significant to understand the electrodynamics of this region because the electron density distribution over the entire low latitude region is controlled by the F-region plasma drift over the equatorial region. Although extensive studies were carried out on the equatorial ionospheric drifts over other longitudes, ${ }^{(1-3)}$ no such studies were reported from India until 1980's, except 
some studies using limited number of days ${ }^{(4,5)}$. In this context, the HF Doppler studies are very vital, as they can provide good insight into ionospheric plasma drift measurements, in particular, and its link with many of the unique geophysical processes, over Indian longitudinal sector.

Since the establishment of Indian Space Research Organization (ISRO) in 1969, scientists were continuously monitoring different aspects of equatorial/low latitude ionospheric phenomena such as Equatorial Electrojet, Equatorial Spread $\mathrm{F}$ and Equatorial Ionization Anomaly using several instruments such as Ionosonde, VHF radar, GPS receivers, magnetometers and many of the low earth orbiting satellites. A few years later, University of Kerala emerged as a Space Science group, supporting the main activities of ISRO. In the year 1982, HF Doppler radar was developed in the University ${ }^{(6)}$ under the ISRO RESPOND programme and operated with support from University Grants Commission (UGC). This was primarily intended for the studies of $\mathrm{F}$ region dynamics with emphasis on Pre-Reversal Enhancement (PRE) during the post sunset period. This radar was operated in the spaced-receiver configuration mode, and could determine velocity vector of the plasma drift. The two horizontal components such as East-West and North-South of the plasma drifts are derived through relative time delays of the Doppler signatures recorded at three receiving points, while the Doppler itself provides the vertical component.

Using the basic single receiver mode of operation, extensive observations have been made on the vertical plasma drifts ${ }^{(7,8)}$. Nearly after two decades, i.e., in 2003 new multi frequency radar was installed. Initially only a vertical sounding facility was installed with one transmitting and one receiving antenna.Two more antennas - one for zonal and another for meridional drift measurements were installed in the second phase during 2006. Both single frequency and multi frequency provided invaluable information about the plasma drifts of the ionosphere during pre sunrise and post sunset periods. The following sections provide the technical details of single and multifrequency radar, method of evaluation of plasma drift and the important scientific contributions came out using the HF Doppler Radar over equatorial region of Thiruvananthapuram in the Indian longitudinal sector

\section{Technical details of HF Doppler radar}

\subsection{Single frequency Radar}

It is a simple system for gaining information on ionospheric plasma motion. The radar system has a wideband pulse transmitting antenna having peak power of $3 \mathrm{~kW}$ and a frequency synthesizer with master oscillator having stability better than one part in $10^{7}$. It also consists of a set of three phase-coherent receivers, a delay counter unit, a multi-channel chart recorder and antennas for transmitting and receiving signals. The transmitter RF, the gate pulses the receiver injection frequencies, and the modulation are all derived from a master synthesizer. So the system is phase coherent. To probe the ionosphere in the bottom side F-region, it is operated at $5.5 \mathrm{MHz}$ with a pulse width of $100 \mu$ s and a pulse repetition frequency (PRF) of $50 \mathrm{~Hz}$. The output RF pulse is fed through a $600 \Omega$ transmission line to a $600 \Omega$ folded dipole for transmission. The transmitted signals are reflected from the equatorial ionosphere and then it is received by three half-wave dipoles located at the comers (Central, North and West) of a right- angled triangle with antenna spacing of $100 \mathrm{~m}$. The signals from the antennas are carried by three identical phase coherent receivers. The phase coherent receivers, each having quadrature channels of $80 \mathrm{~dB}$ gain, detect the ionospheric signal and provide sampled outputs of $A \cos \theta$ and $A \sin \theta$, where $A$ and $\theta$ are the instantaneous amplitude and phase of the received signal.

\subsection{Multifrequency radar}

The multi-frequency HF Doppler radar is a pulsed coherent and monostatic system, which operates at three frequencies- 2.5 $\mathrm{MHz}, 3.5 \mathrm{MHz}$ and $4.5 \mathrm{MHz}$. It can work both in single-frequency (any one frequency of the three) and in multi-frequency modes. The system was fully concept-designed, assembled and installed at the University of Kerala. The transmitter, receivers and antenna system were procured from M/s. Tomco Electronics, Australia. Assembling of systems, installation of antennas, testing, development of hardware and software for data acquisition and analysis were done internally. The time resolution for single frequency operation is fixed at one minute but it can be programmed to get higher resolution. There is a sequential switching scheme for multi-frequency operation, in such a way that the system is operated in one frequency for one minute and after a delay of one minute the next frequency is operated for the next minute duration. The one minute delay in between two frequencies is required for the manual changing of the frequency settings. Thus it can give nearly simultaneous plasma drift data at two different heights with time. Again after one minute, one more height can be probed with the third frequency. This operation can be repeated to get three altitude drift profiles. The recodf8rded drift velocity has to be corrected for contribution due to chemical loss/production mechanisms because on some of the days, layer decay is prominent due to the reflection height of below $300 \mathrm{~km}^{(9)}$. $^{(10)}$ made a comprehensive study to quantify the effects of loss or production in the evening and morning 
time plasma drifts. The technical specifications used for both single frequency and Multifrequency Radar are summarized in Table 1.

Table 1. Technical specifications of single and multifrequency radar

\begin{tabular}{lll}
\hline Type of radar & $\begin{array}{l}\text { HF Doppler system in the spaced-receiver configura- } \\
\text { tion.(Single frequency) }\end{array}$ & $\begin{array}{l}\text { Monostatic Pulsed Coherent HF Doppler radar( } \\
\text { Multi frequency) }\end{array}$ \\
\hline Frequencies of operation & $5.5 \mathrm{MHz}$ & $2.5 \mathrm{MHz}, 3.5 \mathrm{MHz}, 4.5 \mathrm{MHz}$ \\
Peak power output & $3 \mathrm{KW}$ & $5 \mathrm{~kW}$ \\
PRF & $50 \mathrm{~Hz}$ & $100 \mathrm{~Hz}$ (programmable) \\
Pulse width & $100 \mu \mathrm{s}$ & $20 \mu \mathrm{s}$ (programmable) \\
Antenna Type & Three half-wave dipoles & $\begin{array}{l}\text { Three element folded dipole } \\
\text { Receivers }\end{array}$ \\
& Phase coherent and capable of Quadrature detection & $\begin{array}{l}\text { Phase coherent and capable of Quadrature detec- } \\
\text { tion }\end{array}$ \\
A/D converter & Chart recorder, Sense detector & 12 -bit, 100 MHz, 16 channel \\
Number of FFT points & NA & 1024 for each channel \\
Drift data resolution & 5 minute & 1 minute (programmable) \\
\hline
\end{tabular}

\section{Evaluation of vertical Plasma Drifts by Radar}

The vertical plasma drift, which is actually due to the horizontal electric filed is measured from the Doppler shifted reflected echoes. The recorded data from Doppler radar are Fourier analyzed for getting the prominent Doppler frequency $\left(\mathbf{f}_{D}\right)$ corresponding to up or down F-region movement. The vertical plasma drift velocity is calculated using the relation, $\mathrm{Vz}=-$ $\left(f_{D} \times \lambda\right) / 2$, where $\lambda$ is the sounding wavelength. These drifts actually correspond to the plasma motion over $\mathrm{F}$ region altitudes from which the reflected echoes are coming. This reflection height is measured from the delay of time between the transmitted signal and the echo for each operating frequency, which has a resolution of $1.5 \mathrm{~km}$. The radar is capable of recording vertical drift data in the evening-to-morning sector between 17:00 $\mathrm{hr}$ LT and 08:00 $\mathrm{hr}$ LT with 1 minute data resolution. However, the measured drift velocity has to be corrected as it includes the contribution due to chemical loss/production mechanisms. This is to be considered because on some of the days, the reflection height is below $300 \mathrm{~km}$ where layer decay is prominent ${ }^{(9)}$. Therefore in a seminal work, ${ }^{(10)}$ have made a comprehensive study to quantify the effects of loss/production in the evening/morning time vertical plasma drifts. They found that these corrections can indeed be as high as $10-15 \mathrm{~m} / \mathrm{s}$ and have strong altitude as well as seasonal dependence. The increase in the apparent drift with height has been attributed to the exponential decrease in the neutral density of $\mathrm{O}_{2}$ and $\mathrm{N}_{2}$, while the seasonal variations were accounted for by the atmospheric expansion/compression during summer/winter months. TTherefore, proper corrections to the observed vertical plasma drift are well apprehended for the realistic interpretation of the data.

\section{Scientific results from Single Frequency Radar}

The first ever report on the vertical plasma drift measurements using the HF Radar measurements over Trivandrum was published by ${ }^{(7)}$. The studies presents preliminary observations on the prereversal enhancement (PRE) in vertical plasma drifts using 15 days of data during the period January 21 to April 25, 1984, and found that under moderately disturbed conditions, the enhancement in vertical plasma drift was down by a factor of two, in comparison with highly disturbed conditions. The pattern of drift velocity dependence on magnetic activity was observed to be consistent with that of spread F occurrence. Following this ${ }^{(8)}$ studied the post-sunset equatorial F-region at Thiruvananthapuram, over various ranges of solar and geomagnetic conditions. The study revealed that under quiet magnetic activity conditions, PRE is quite sensitive to solar activity during the two vernal equinoxes of 1984 and 1985. They have attributed the effects to the increased plasma density during the solar maxima associated with the changes in solar radiation and thermospheric temperature. In another study ${ }^{(11)}$ have used a large set i.e., 137 days of vertical plasma drifts data which span equally among different seasons during the years 1984-1986. Their observations showed constant diurnal variation and the drift velocities were directed upward/downward during day/night. In addition pre reversal enhancement of vertical drift was found to exhibit seasonal, solar, and magnetic activity effects. They could see that during high solar activity, the average peak velocity of the enhancement has dropped from $\sim 40 \mathrm{~m} \mathrm{~s}^{-1}$ in equinox to $\sim 10 \mathrm{~m} \mathrm{~s}^{-1}$ in summer. The observations of the dependence of occurrence of ESF on magnetic activity very well match with observations by $^{(12)}$. They compared their results with the observations from Jicamarca ${ }^{(13,14)}$ to bring out the major longitudinal differences, 
if any. The spectra of fluctuations in the post sunset $\mathrm{F}$ region was studied by ${ }^{(15)}$. They have noticed that the fluctuations consist of a number of components with periodicities varying from a few minutes to several tens of minutes. The dominant fluctuations were found to have periods of the order of a few tens of minutes with the most frequent being about $30 \mathrm{~min}$.

When compared the observations with Jicamarca radar data, where perturbations were found to have largest amplitudes in the midnight-sunrise period ${ }^{(16)}$, Thiruvananthapuram data revealed largest amplitudes at around the evening PRE time. The long duration (several hours) perturbations seen at Jicamarca were not seen at Thiruvananthapuram also. These changes were mainly attributed to the data confinement and the use of single operating frequency of the HF Doppler system at Thiruvananthapuram.

In fact, ${ }^{(17)}$ recorded the time variations of the vertical drift velocity, their mean values and mean group height of the reflecting layer for eight days, during the afternoon and midnight period of March - April 1988. The observed plasma drift velocity, east-west plasma drift velocity and north -south plasma drift (representing meridional wind) velocity, were consistent with the earlier observations from the same radar and the mechanism can be well explained by F-region dynamo theory. In another important work, ${ }^{(18)}$ simultaneously observed F-region vertical plasma drift during evening hours (1700-2100 IST) at Thiruvananthapuram $\left(\operatorname{dip} 0.6^{\circ} \mathrm{N}\right)$ and Kodaikanal $\left(\operatorname{dip} 4^{\circ} \mathrm{N}\right)$ for fifteen days during winter solstice and solar minimum conditions. Even though, the F-region vertical velocity due to zonal electric field and its temporal pattern is found to be more or less the same at Thiruvananthapuram and Kodaikanal on all the days as expected due to the proximity of equator at both locations, the magnitude of drift was different at the two stations on all the days testifying to the presence of height gradients in vertical plasma drift and an altitude dependence of the velocity gradient.

The observed results are attributed to the altitudinal dependence of $\mathrm{E}$ and $\mathrm{F}$ region dynamos over low and equatorial latitudes. It was suggested that the net result of this altitude dependence would be the development of vertical shear in zonal plasma drift which was widely observed over other longitudes ${ }^{(3)}$. So the reported decay of positive height gradient in vertical plasma drift in the lower F region (Thiruvananthapuram) could be another signature of this physical situation.

The enigmatic phenomena of Equatorial Spread-F and its imprints on the HF Doppler Radar measurements had also been attempted in an earlier study ${ }^{(19)}$. They analyzed the spectral width, correlation lifetime, along with vertical electromagnetic drift velocity of spread-F irregularities and observed an inverse relationship between intensity (spectral width) and life time of spread $\mathrm{F}$ irregularities. They also found that regions of high echo power are associated with low correlation lifetime and vice versa. The observed vertical plasma drift velocity during spread-F showed a complicated pattern, with a change in sign every few minutes with magnitude. They attributed this effects to the existence of unexpectedly large drift velocity to the mechanism capable of producing turbulent electric field having r.m.s. value several times higher than the normal dynamo electric field. The necessary condition for this mechanism had been justified on the basis of low electron concentration in the lower ionosphere near the equator at night and in the early part of the sunrise period.

In another work ${ }^{(20)}$ had reported a strong seasonal dependence of vertical plasma drift (Vd) and pronounced PRE during most of the spread F days. They compared the results with Jicamarca data and found a good agreement with the observation. The results were interpreted on the basis of the pronounced F-region dynamo effects, which made it conducive for the occurrence of Spread-F. The magnetic activity dependence of Vd and its influence on the occurrence of ESF had also been attempted in literature ${ }^{(21)}$ using the data during 1994-1995, They analyzed the magnetic activity and seasonal dependence on the percentage occurrence of spread $\mathrm{F}$ and showed that magnetic activity was not a significant factor in deciding the onset of spread $\mathrm{F}$. But at the same time the occurrence pattern of ESF was found to have a strong seasonal dependence with summer minimum which was in agreement with previous studies ${ }^{(5)}$.

A very interesting feature of $\mathrm{F}$ region i.e., signatures of plasma velocity vortex was observed by ${ }^{(22)}$. The resultant plasma drift velocity vector in the vertical zonal plane was found to exhibit a gradual rotation in the evening time which proved existence of velocity vortex during post sunset period. This indicated the presence of an evening curl free $\mathrm{F}$ region electric field corroborating many of the earlier results ${ }^{(23)}$. The inverse relation of mean frequency of the radar measured velocity fluctuations with magnetic activity index $\boldsymbol{A}_{p}$ was brought out in another study using limited number of observations ${ }^{(24)}$. In fact, the study should be extended with more number of days to understand the cause-and effect relation between these two parameters.

Although the afore-mentioned results have substantially improved our understanding of equatorial vertical plasma drift and its role in modulating the processes in the F region, they were constrained by the probing of single frequency, i.e., $5.5 \mathrm{MHz}$. In order to have a comprehensive understanding from different altitude regions of the F region, a Multi frequency mode of operation was proposed and hence the system had been updated to Multi frequency mode since 2003 onwards.

\section{Scientific Results from Multifrequency Radar}

As mentioned earlier, multi frequency Radar can work both in single-frequency (any one frequency of the three) and in multifrequency modes (2.5 MHz3.5 MHz and 4.5 MHz). This radar was operational until 2010, and many important studies have 
been carried out to examine various aspects such as temporal evolution and height gradient of vertical plasma drift, meridional wind observations, electrodynamical behavior of ionosphere during pre-sunrise period, signature of velocity vortex, vertical shear at the $\mathrm{F}$ region, periodicities in the Vd during magnetically quiet and disturbed conditions etc.

(25) came up with preliminary results from this radar. Other than the primary observation of day to day variation in Vd, they carried out the studies regarding the seasonal variation of PRE in Zonal electric field using a sounding frequency of 3.5 MHz. They could see that as the season advances from summer to equinox, the magnitude of Vd increases and again from equinox to winter $\mathrm{Vd}$ decreases for 70 days of observation. These changes were attributed to the variation of strength of $\mathrm{F}$ region dynamo with season. The peaking magnitude of Pre Reversal Enhancement (PRE) during equinox was found to match with the earlier observations using single frequency radar by ${ }^{(8,11)}$ with the observed solar activity dependence of Vd was attributed to the increase in equatorial zonal wind, and the ratio of field line Pederson conductivities in E and F region.

Height gradient in vertical plasma drift using HF Radar was analyzed by ${ }^{(26)}$. Their analysis revealed that negative value around PRE time followed by a positive after the reversal. Their observations were in good agreement with the model prediction of $^{(27)}$ supporting the curl-free or irrotational nature of equatorial zonal electric fields. Also they could see that the magnitude of the negative height gradient of vertical plasma drift decreases as the probing altitude increases below the F peak because as the altitude of the $\mathrm{F}$ region is higher, the magnetic flux tubes maps to the conjugate $\mathrm{E}$ region at higher latitudes where the separation between the magnetic meridian and the sunset terminator is larger. The seasonal variation in the height gradient around the PRE was explained on the basis of seasonal variation of the alignment of the solar terminator with respect to the magnetic field lines.

Another important contribution from the HF radar was the determination of the meridional wind component of plasma drift. ${ }^{(28)}$ investigated this aspect using the radar operated at $5.5 \mathrm{MHz}$, by calculating the time delay between the signals at the north and central antenna of the radar by cross correlation method. The temporal variation of these wind data was found to have good agreement with the ionosonde derived winds using h'F data. The results were also compared with horizontal wind model HWM93, and were in good agreement with that except during pre-reversal and post-reversal period as reported by ${ }^{(29)}$.

(30) investigated the signatures of velocity vortex using the Multi frequency HF Doppler data. They showed a downward excursion of plasma drift having a duration of $\sim 30$ minutes near the sunrise followed by an upward drift beyond 06:30 LT. This feature of plasma drift is similar to the post sunset enhancement followed by the reversal in plasma drift direction. So there exists an enhancement in the vertical drift in the negative direction before sunrise similar to a positive enhancement in vertical drift after sunset.

Following this, the pre-sunrise equatorial F-region dynamics was investigated in detail using digital Ionosonde and HF Doppler radar ${ }^{(31)}$. They have explained these changes using unique electrodynamics for the establishment of a curl free nature of zonal electric field over day/night time equatorial ionosphere. The spectral variability of vertical plasma drift has also been attempted ${ }^{(32)}$ using wavelet analysis. The observed fluctuations having periodicities in the range 4-32 minutes were attributed to the atmospheric gravity waves. Later ${ }^{(33)}$ have studied the periodicities in Vd during geomagnetically quiet and disturbed days. They could see that $\mathrm{V}_{d}$ on geomagnetically quiet days have a periodicity of 15-20 minutes, whereas on disturbed days it exhibits $\sim 45$ minutes periodicity,

It has also been observed that whenever post sunset $\mathrm{V}_{d}$ shows a periodicity of 15-20 minutes the equatorial spread-F occurs. The presence of a vertical shear in zonal drift and its altitude dependence was observed by ${ }^{(34)}$. The shear region was found to be in the altitude range of 200-300 km where the F and $\mathrm{E}$-region compete for dominance. The negative gradient in the vertical drift and shear in the zonal drift again proved that evening equatorial ionosphere maintains the curl-free nature of the electric field which was supported by earlier studies ${ }^{(22)}$.

\section{Current status and future scope}

From the above discussion we can see that HF Doppler Radar is an excellent facility and when operated in the spaced-receiver configuration, provided wealthy of information on the ionospheric F region dynamo, particularly during the post sunset period. It can also be used to find the behavior of the F region during the pre-sunrise period in addition to the thermospheric zonal/meridional plasma drift characteristics. In fact, characteristics of plasma velocity vortex also remain as one of the least explored aspects using this radar, which are expected to have significant implications in the current understanding of solarterrestrial research. Unfortunately this unique research facility of the University of Kerala was not operational since 2010. It was damaged due to the unexpected lightning problem. The frequency Synthesis Unit (FSU) was seriously affected by the lightningstrike, which could not be rectified so far. However, it must be mentioned that some of the results reported using this Radar are quite unique. They have not only improved our understanding of evening/morning time equatorial $\mathrm{F}$ region, but also provided new direction to many of the future studies having significant implications. To the best of our knowledge, currently there are no such facilities in India, which are exclusively used for ionospheric probing and academic research. In this context, we believe 
that a new HF Doppler Radar with extended facility is highly essential for the growth of research and development activities, particularly for the Colleges and Universities in India. Although the prime objective of this work is to review the importance of earlier studies using the HF Doppler radar, a few observations using this radar are also described here for completeness.

Figure $1(\mathrm{a}-\mathrm{c})$ shows the time variation of vertical plasma drift observed during evening hours over Trivandrum using multi frequency HF Doppler Radar at 2.5, 3.5 and 4.5 MHz respectively. From the figure it is clear that vertical velocity shows a clear pre-reversal enhancement around 18:30 IST, which is a characteristic F region phenomenon during the sunset period over dip equatorial ionosphere. The error bars actually show the contribution of chemistry and are found to be maximum before 18:00 IST and after 21:30 IST. This is the typical pattern over Trivandrum. The increased values of velocity due to chemical loss are an indicative of lowering of reflection height during these periods.

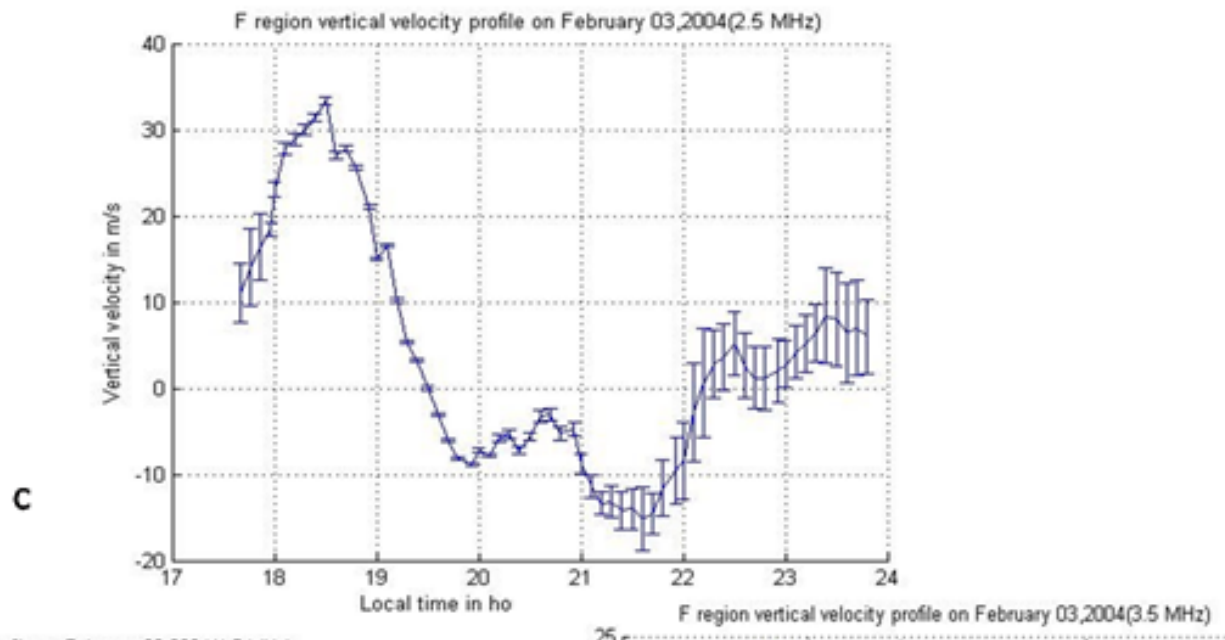

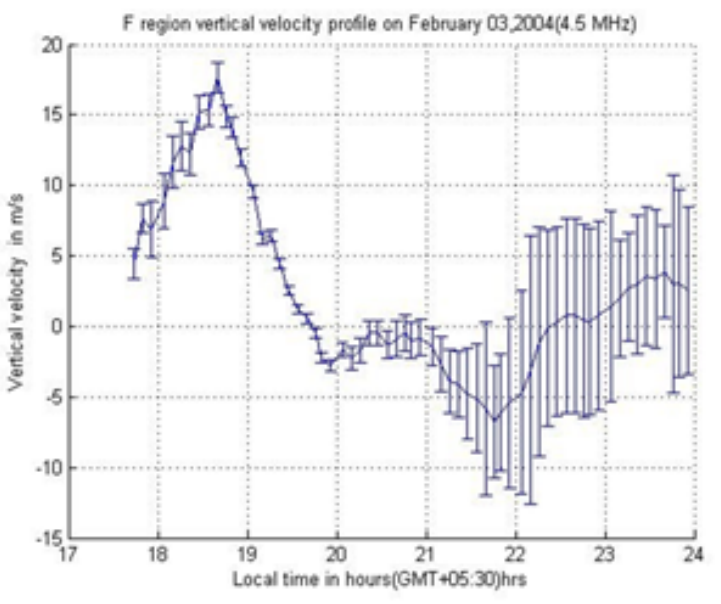

a

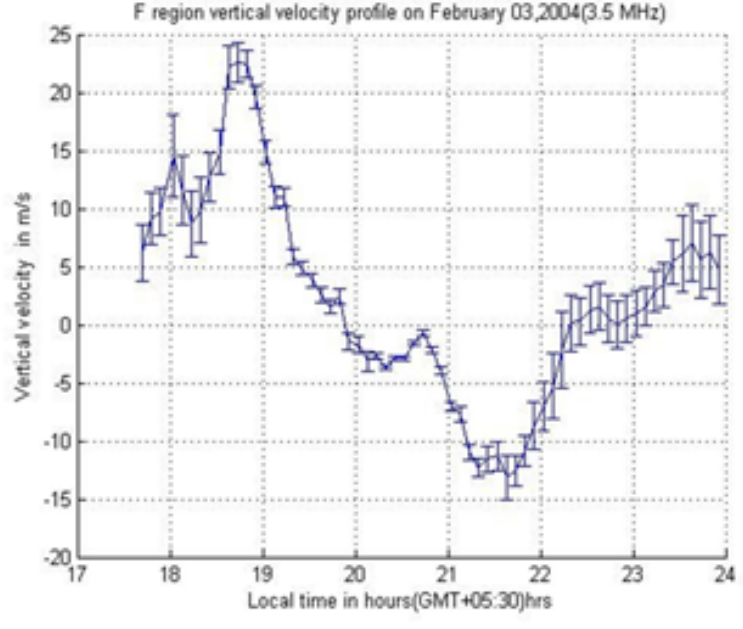

b

Fig 1. (a-c): Typical vertical velocity variation of February 04, 2004, showing Pre-Reversal Enhancement during post sunset time.

In addition to the plasma drift during evening hours, similar behavior is observed in the negative direction during morning hours. Figure $2(\mathrm{a}-\mathrm{c})$ shows the time variation of vertical plasma drift observed during morning hours over Trivandrum using multi frequency HF Doppler Radar at 2.5, 3.5 and $4.5 \mathrm{MHz}$ respectively. It is clearly seen that similar to the pre reversal enhancement, a sunrise downward enhancement (SDE) is observed at around 6:30 IST. This aspect has been addressed in many of the earlier works ${ }^{(30,32)}$. These sorts of observations provided many unique results concerning the electrodynamical behavior of the ionosphere during morning hours. During morning hours, the plasma drifts due to chemical production are shown using error bars and are found to be maximum after 5:30 IST. The details regarding the altitudinal and seasonal variations in the plasma drift due to chemical loss and production have also been addressed comprehensively in ${ }^{(10)}$, as mentioned earlier. 


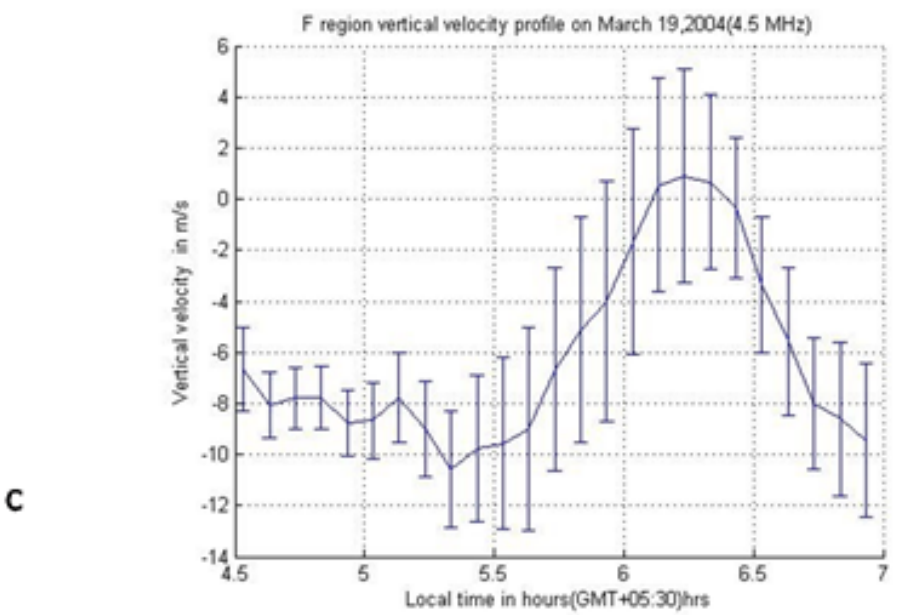

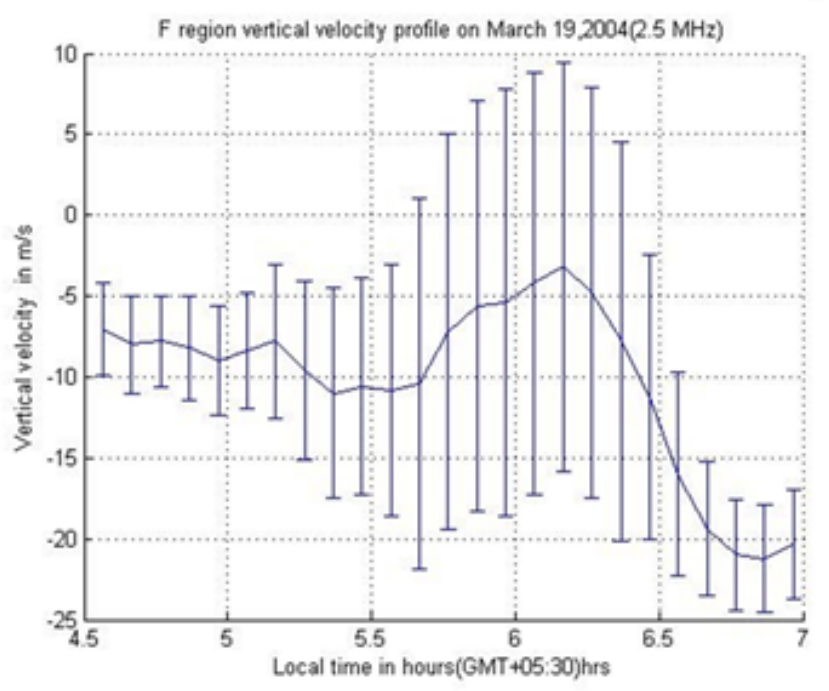

a

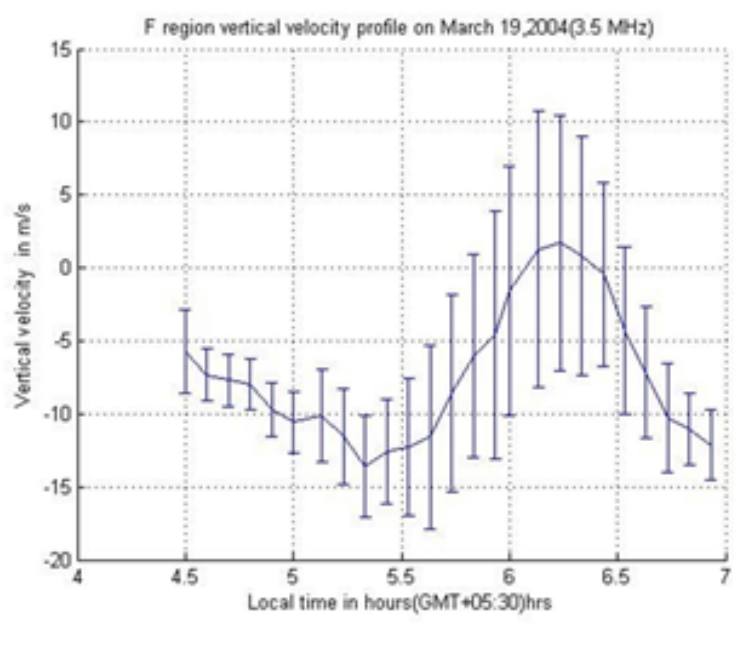

b

Fig 2. (a-c): Typical vertical velocity variation of February 04, 2004, showing Pre-Reversal Enhancement during presunrise time.

The F region vertical velocity profiles of March 19, 2004 during morning hours between 04:30 LT and 07:00 LT at 2.5 MHz, 3.5 $\mathrm{MHz}$ and $4.5 \mathrm{MHz}$ along with the corrected profiles are shown in the Figure $3(\mathrm{a}-\mathrm{b})$. Even though the observation is insufficient of obtaining the Sun rise Downward Enhancement (SDE) completely, it gives us a signature of it after 06:45 LT. An upward enhancement in vertical drift is noted just before SDE. This can be due to the production of an additional layer at the top of F-region due to sunrise and subsequently the newly formed layer supersedes the old layer. It is seen that the drift in this sector is positive incorporating the production effect; otherwise it is observed to be negative. Thus the observed vertical plasma drift is attributed to the combined effect of vertical electrodynamic effect and the vertical drift due to chemical production arising from changes in the reflection height. The features of the vertical drifts on this morning after chemical correction are given in Figure $3 \mathrm{~b}$. We can see that it shows a clear distinction from Figure 3a. All the three frequencies show a positive vertical drift value as compared to the Figure 3a. From the above discussion it is evident that chemical loss or production effects must be considered while interpreting many of the HF Doppler observations. We believe that similar morning time observations can have significant implications in the understanding of equatorial electrodynamics of the F region comprehensively. 


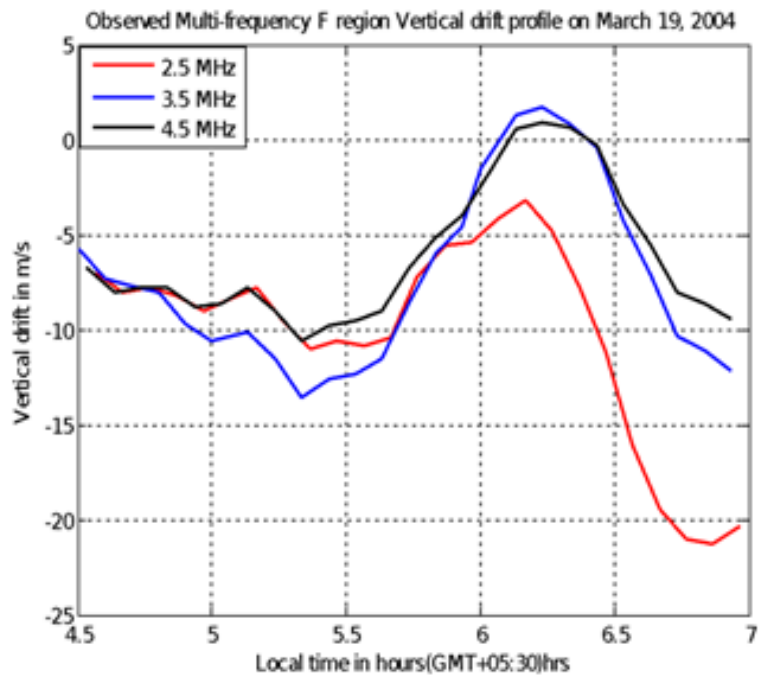

a

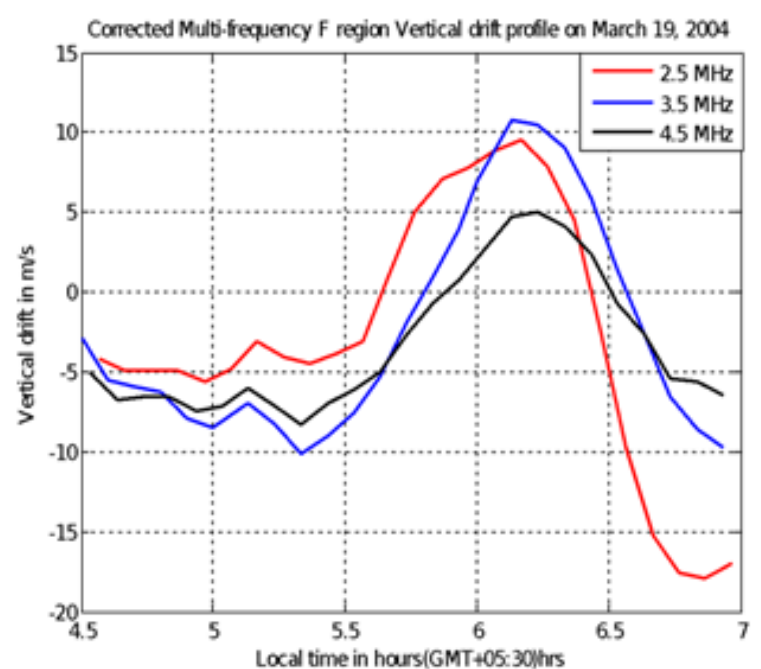

b

Fig 3. (a-b) .(a) The observed Multi-frequency sounding Vertical Velocity profile on the early morning of March 19, 2004 and (b)after applying the chemical contributiondue to production.

In this context, it is to be noted that nearly about 40 research papers have been published in reputed national and international journals using this Radar data. The details of year wise publication are presented in Figure 4. In view of the above, we plan to propose a new state-of-the-art Radar facility over Trivandrum, in near-future, in collaboration with various Universities in Kerala, ISRO and other Government organizations such as DST (Department of Science and Technology), UGC and KSCSTE (Kerala State Council for Science, Technology and Environment). We believe that the system would not only provide a definite route map for the future fundamental level academic research activities in India, but also be helpful in improving the reliability of communication/navigation systems thereof.

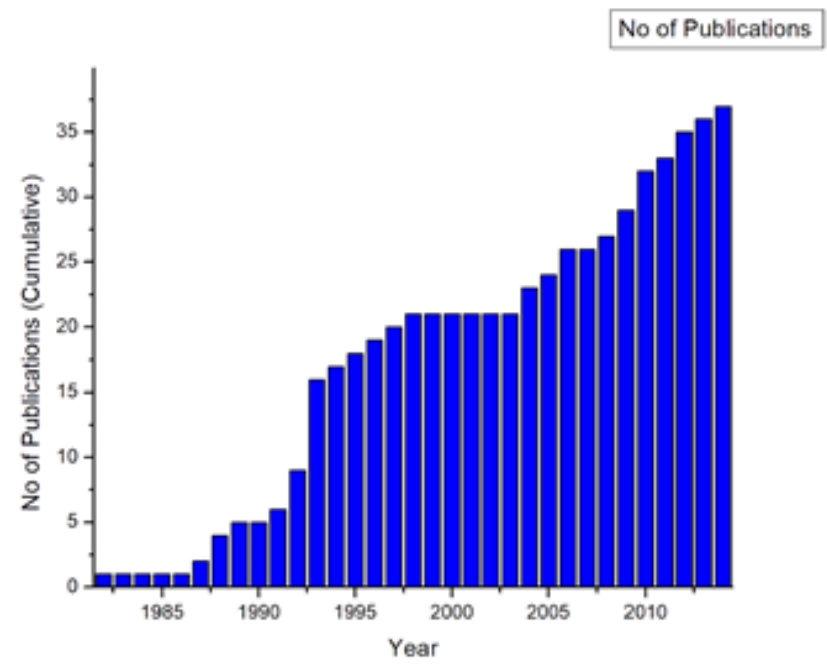

Fig 4. Year wise details of number of publications from the HF Doppler Radar 


\section{References}

1) Woodman RF. Vertical drifts and east-west electric fields at the magnetic equator. J GeophysRes;75(31):6249-6259. Available from: https://doi.org/10. 1029/JA075i031p06249.

2) Fejer BG, Gonzales CA, Farley DT, Kelley MC, Woodman RF. Equatorial electric fields during magnetically disturbed conditions 1 . The effect of the interplanetary magnetic field. Journal of Geophysical Research. 1979;84(A10). Available from: https://dx.doi.org/10.1029/ja084ia10p05797.

3) Fejer BG, Kudeki E, Farley DT. Equatorial F region zonal plasma drifts. Journal of Geophysical Research. 1985;90(A12). Available from: https: //dx.doi.org/10.1029/ja090ia12p12249.

4) Ramesh KB, Sastri JH. Solar cycle and seasonal variations in F-region vertical drifts over Kodaikanal, India. Annales Geophysicae. 1995;13(6):633-640. Available from: https://dx.doi.org/10.1007/s00585-995-0633-7.

5) Subbarao KSV, Murthy BVK. Post-sunset F-region vertical velocity variations at magnetic equator. J Atmos Terr Phys. 1994;56(1):59-65. Available from: https://doi.org/10.1016/0021-9169(94)90176-7.

6) Balan N, Murthy K, Reddi CR, Rao PB, and KSVS. Proc Indian Natl Sci Acad. vol. 48. 1982. Available from: https://doi.org/10.1016/0021-9169(92)90162-E.

7) Jayachandran B, Balan N, Nampoothiri SP, Rao PB. HF Doppler observations of vertical plasma drifts in the evening F region at the equator. Journal of Geophysical Research. 1987;92(A10). Available from: https://dx.doi.org/10.1029/ja092ia10p11253.

8) Namboothiri SP, Jayachandran B, Balan N, Rao PB. Vertical plasma drifts in the post-sunset F-region at the magnetic equator. Journal of Atmospheric and Terrestrial Physics. 1988;50(12):1087-1091. Available from: https://dx.doi.org/10.1016/0021-9169(88)90097-9.

9) Bittencourt JA, Abdu MA. A theoretical comparison between apparent vertical velocities and real vertical E B plasma drift velocities in the equatorial F-region. JGeophysRes. 1981;86(A4):2451-2454. Available from: https://doi.org/10.1029/JA086iA04p02451.

10) Sumod T, Pant, Nayar S. On the variability of the observed HF Doppler derived equatorial F-region plasma drifts during evening and morning hours and the corrections therein. Ind J Radio And Space Phys. 2012;41:130-130. Available from: http://hdl.handle.net/123456789/14040.

11) Namboothiri SP, Balan N, Rao PB. Vertical plasma drifts in the F region at the magnetic equator. Journal of Geophysical Research. 1989;94(A9). Available from: https://dx.doi.org/10.1029/ja094ia09p12055.

12) Somayjulu VV, Murthy K. Nature of association of equatorial spread F with magnetic activity. Nature. 1976;263:36-37. Available from: https: //www.nature.com/articles/263036a0.

13) Fejer BG, Farley DT, Woodman RF, Calderon. Dependence of equatorial F region vertical drifts on season and solar cycle. J Geophys Res;p. 5792-5796. Available from: https://doi.org/10.1029/JA084iA10p05792.

14) Woodman RF, Rastogi RG, Calderon C. Solar cycle effects on the electric fields in the equatorial ionosphere. J Geophys Res. 1997;82:5257-5261. Available from: https://doi.org/10.1029/JA075i031p06249.

15) Nair RB, Balan N, Bailey GJ, Rao PB. Spectra of the ac electric fields in the post-sunset F-region at the magnetic equator. Planetary and Space Science. 1992;40(5):655-662. Available from: https://dx.doi.org/10.1016/0032-0633(92)90006-a.

16) Fejer BG, Larsen MF, Farley DT. Equatorial disturbance dynamo electric fields. Geophysical Research Letters. 1983;10(7):537-540. Available from: https://dx.doi.org/10.1029/g1010i007p00537.

17) Balan N, Jayachandran B, Nair RB, Namboothiri SP, Bailey GJ, Rao PB. HF Doppler observations of vector plasma drifts in the evening F-region at the magnetic equator. Journal of Atmospheric and Terrestrial Physics. 1992;54(11-12):1545-1554. Available from: https://dx.doi.org/10.1016/0021-9169(92) 90162-e.

18) Sastri JH, Varma VKM, Nayar SRP. Height gradient of F region vertical drift in the evening equatorial ionosphere. Geophysical Research Letters. 1995;22(19):2645-2648. Available from: https://dx.doi.org/10.1029/95gl02668.

19) Joymon D, Solomon S, Nayar SR. HF Doppler radar observations on the relationship between electrostatic turbulence and equatorial spread-F. Ind $J$ Radio And Space Phys . 1996a;25:340-344. Available from: http://nopr.niscair.res.in/handle/123456789/35680.

20) Joymon D, Solomon S, George TM, Meena VK, R NS. Observation of day-to-day and seasonal variability in vertical F-region plasma drift at magnetic equator using HF Doppler radar. Ind J Radio And Space Phys. 1996;25:158-162. Available from: https://www.researchgate.net/publication/286896902.

21) Joymon D, Solomon S, George TM, Nayar SR. Magnetic activity dependence of vertical plasma drift and its influence on spread-F. Ind J Radio And Space Phys. 1997;26:213-217. Available from: http://nopr.niscair.res.in/bitstream/123456789/35522/1.

22) Sreehari CV, Bhuvanendran C, Nayar S. HF Doppler radar observations of vertical and zonal plasma drifts-Signature of a plasma velocity vortex in evening F-region. Ind J Radio And Space Phys. 2006;35. Available from: https://www.researchgate.net/publication/254212982.

23) Kudeki E, Bhattacharya S. Post sunset Vortex in equatorial F region plasma drifts and implications for bottom side Spread F. J Geophys Res. 1999;101:2816328163. Available from: https://doi.org/10.1029/1998JA900111.

24) George T, Joymon D, Solomon S, Rajesh P, Nayar S, Revathy K. Fluctuations in vertical plasma drift and magnetic activity. J Atmos Terr Phys. 1998;27:233235. Available from: http://nopr.niscair.res.in/handle/123456789/35314.

25) Nayar SR, Sreehari CV, Shibu S, Devassia C, Rao KSV, Mohankumar SV. Multi-frequency HF Doppler radar observations of vertical plasma drift Preliminary results . Ind J Radio And Space Phys. 2005;34:233-242. Available from: http://hdl.handle.net/123456789/19319.

26) Nayar SRP. Investigation of height gradient in vertical plasma drift at equatorial ionosphere using multifrequency HF Doppler radar. Journal of Geophysical Research. 2004;109(A12). Available from: https://dx.doi.org/10.1029/2004ja010641.

27) Murphy JA, Heelis RA. Implications of the relationship between electromagnetic drift components at mid and low latitudes. Planetary and Space Science. 1986;34(7):645-652. Available from: https://dx.doi.org/10.1016/0032-0633(86)90042-5.

28) Nayar SR, Bhuvanendran C, Jyoti N, Devasia CV, Subbarao KSV. Meridional wind derived from HF doppler radar and ionosonde over the magnetic equator. Ind J Radio And Space Phys. 2004;33:367-372. Available from: http://hdl.handle.net/123456789/19341.

29) Igi S, Ogawa T, Oliver WL, Fukao S. Thermospheric winds over Japan: Comparison of ionosonde and radar measurements. American Geophysical Union (AGU). 1995. Available from: https://dx.doi.org/10.1029/95ja02387. doi:10.1029/95ja02387.

30) Tiju M, Nayar S, Sudha R, Tarun P. Electrodynamics of the Equatorial F region ionosphere using Post-sunset and Pre-sunrise Vertical Plasma drift Observations . In: and others, editor. 37th COSPAR Scientific Assembly. 2008. Available from: https://ui.adsabs.harvard.edu/abs/2008cosp...37.1407M/ abstract.

31) Nayar SRP, Mathew TJ, Sreehari CV, Sumod SG, Devasia CV, Ravindran S, et al. Electrodynamics of the equatorial F-region ionosphere during pre-sunrise period. Annales Geophysicae. 2009;27(1):107-111. Available from: https://dx.doi.org/10.5194/angeo-27-107-2009.

32) Mathew TJ, Nayar SRP, Ravindran S, Pant T. Characteristics of the equatorial F-region vertical plasma drift observed during post-sunset and pre-sunrise hours. Advances in Space Research. 2010;46(5):618-625. Available from: https://dx.doi.org/10.1016/j.asr.2010.04.023. 
33) Simi KG, Vineeth C, Pant TK, S A. Analysis of the Vertical Drifts in the Post Sunset Equatorial F-region during Geomagnetically Quiet and Disturbed periods. Ind J Radio Space Phys. 2013;42:229-239. Available from: http://hdl.handle.net/123456789/20915.

34) Tiju M, Nayar S. Characteristics of zonal plasma drift during post-sunset hours observed using multifrequency HF Doppler radar .38th COSPAR Scientific Assembly. In: and others, editor. 38th COSPAR Scientific Assembly. 2010. 